Urologe 2019·58:59-60

https://doi.org/10.1007/s00120-018-0834-9

Online publiziert: 19. Dezember 2018

(c) Springer Medizin Verlag $\mathrm{GmbH}$, ein Teil von Springer Nature 2018

CrossMark

Michael Stephan-Odenthal

Urologie Rhein Berg, Leverkusen, Deutschland

\title{
Urologie im Jahr 2025 - Sicht des Urologen in der Praxis
}

motiviert, das mich seit 2008 in meiner Funktion im Beirat und später in der Geschäftsführung der UroGmbH Nordrhein, im Berufsverband der Urologen seit 2012 und in der Landesärztekammer seit 2015 antreibt.

An erster Stelle stehend ist für mich die freiberufliche selbstbestimmte Tätigkeit als Arzt, trotz aller Veränderungen im Gesundheitswesen, immer noch erfüllend. Der persönliche Bezug zu Patienten und die Gestaltungsfreiheit im Austausch und in der Diskussion mit gleichgesinnten Kollegen sind für mich Grundlage meiner hohen beruflichen Zufriedenheit. Das hohe Maß an Verantwortung gegenüber den Patienten empfinde ich als Bürde aber nicht als Last. Belastend ist lediglich der ständige Kampf gegen immer neue und sich ändernde bürokratische Regeln als Ausdruck der versuchten Fremdbestimmung durch Politik und Kostenträger. Als „Einzelkämpfer“ würde ich diesen nicht bewältigen wollen. An zweiter Stelle ist das wirtschaftliche Auskommen als Anteilseigner eines „Unternehmen Arztpraxis“ immer noch überdurchschnittlich. Das unternehmerische Risiko ist dabei nicht unerheblich, jedoch mit Sachverstand im Team überschaubar. Im Nachhinein würde ich mir bei meinem Start in die Selbständigkeit mehr Kenntnisse in Betriebsführung und Ökonomie gewünscht haben. Dadurch wäre diese Lernkurve kürzer und die Abhängigkeit von Beratern geringer gewesen.

Die Zukunft der Urologie sehe ich trotz einer demographischen Bevölkerungsveränderung, die für die Urologen spricht, mit gemischten Gefühlen. Einer wachsenden Nachfrage nach urologischen Behandlungen und Behandlungsmöglichkeiten stehen langfristig weniger Ärzte und nur unterproportional stei- gende wirtschaftliche Möglichkeiten entgegen. Wir werden als Urologen in Klinik und Praxis immer Beschäftigung und wirtschaftliches Auskommen haben, aber mit immer stärkeren Effizienzanforderungen konfrontiert werden, welche die Zufriedenheit als Arzt belasten werden. Insbesondere wenn diese Effizienzanforderungen wie bisher weiter überwiegend fremd bestimmt werden, ist unser Fachgebiet in Klinik und Praxis gefährdet. Chancen in einer Verbesserung unserer Effizienz sehe ich nur in Verbesserung der Ausbildung weiterer Vernetzung über die Sektorengrenzen hinweg. Derzeit wird die Vernetzung v.a. durch die sektorale Verteilung der Honorare und damit der wirtschaftlichen Grundlage gehemmt. Eine Aufhebung dieser wirtschaftlichen Sektorengrenzen ist zwar von der Politik vorgesehen (mit der ASV gestartet), braucht aber sicher noch viele Jahre der Umsetzung. Pragmatisch sind deshalb über die Sektorengrenzen hinweg derzeit nur Teilarbeitsmodelle von Urologinnen und Urologen oder Belegarztmodelle. Ob solche Modelle in Gemeinschaftspraxen oder MVZ umgesetzt werden, hängt von den freiberuflichen Vorstellungen und Wünschen der Handelnden ab.

Für unser Fachgebiet wünsche ich mir zukünftig lokale Verbünde von Urologen in Klinik und Praxis, die für die lokale Bevölkerung eine qualitative hochwertige urologische Versorgung sicherstellen. In der Gruppe werden persönliche werden persönliche Schwerpunkte je nach Talent und Fähigkeit entwickelt und gepflegt und Verantwortungsbereiche festgelegt. Die jeweiligen Urologinnen und Urologen sind dabei je nach Behandlungsanforderung und Schwerpunkt sowohl in der Klinik als auch in der Praxis zu einem berufspolitischen Engagement 
tätig. Im Verbund werden junge Kolleginnen und Kollegen ausgebildet. Urologische Forschung findet für Interessierte in Rotationsprogrammen an den Universitätskliniken statt. Alle Urologinnen und Urologen beteiligen sich im Rahmen von Registern an der Versorgungsforschung.

Fazit: Die selbständige Tätigkeit in der Praxis ist immer noch eine attraktive und erfüllende Ausübung unseres ärztlichen Berufs mit wirtschaftlich zufriedenstellendem Auskommen. Vorteile sind die Patientenbindung und die gestalterischen Möglichkeiten der eigenen Arbeit. Nachteil ist der Aufwand in Bürokratie. Herausforderungen in Zukunft werden der Erhalt der Freiberuflichkeit und die Ausübung in Gemeinschaft und vernetzten Strukturen über die Sektorengrenzen hinweg sein.

\section{Korrespondenzadresse}

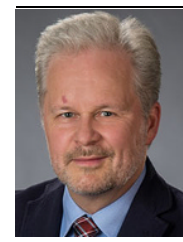

Dr. Michael StephanOdenthal

Urologie Rhein Berg

Am Gesundheitspark 4, 51375 Leverkusen,

Deutschland

dr.stephan-odenthal@

t-online.de

Dr. Michael Stephan-Odenthal ist Facharzt für Urologie mit Zusatzbezeichnung medikamentöse Tumortherapie, Palliativmedizin, Andrologie, urologische Röntgendiagnostik.

\section{Einhaltung ethischer Richtlinien}

Interessenkonflikt. M. Stephan-Odenthal gibt an, dass kein Interessenkonflikt besteht.

Hier steht eine Anzeige. 